\title{
Resiliencia y felicidad de adolescentes frente a la marginación urbana en México \\ Elián Gómez-Azcarate ${ }^{1}$, Alejandro Vera², María-Elena Ávila², Gonzalo \\ Musitu $^{3}$, Enrique Vega² \& Gabriel Dorantes ${ }^{2}$
}

\section{Artículo}

Material original autorizado para la publicación en la revista Psicodebate. Facultad de Ciencias Sociales. Universidad de Palermo.

Recibido 10-02-2014 | Aceptado 14-04-2014

\section{Resumen}

La marginación urbana en México ha crecido enormemente en los últimos años afectando directamente a los jóvenes. En este estudio empírico se analizó su efecto sobre la resiliencia y felicidad juvenil en función de factores estructurales. Se aplicó el Cuestionario RESI-M y la Escala de Felicidad de un ítem, a 1201 estudiantes mexicanos de secundaria y bachillerato. Los resultados mostraron que la marginación afecta de forma diferenciada las dimensiones de la resiliencia. Además, dentro de los grupos por grado de marginación, hubo diferencias significativas en la resiliencia según sexo, escolaridad y tipo de familia. Los análisis de regresión lineal mostraron el valor predictivo de algunos de los factores estructurales. Se observó cómo las condiciones de vida marginales afectan la capacidad de resiliencia de los adolescentes, aunque no la predicen dado que el individuo puede transformarse a sí mismo y a su realidad adversa ya que la felicidad no se asocia a las condiciones del contexto, excepto en relación a la edad y la familia. Se analiza la influencia diferenciada de los tipos de familias en relación a la marginación y su papel en el desarrollo de la resiliencia para afrontar los retos futuros y evitar el desajuste social. Se aportan elementos para profundiza el estudio de los adolescentes en marginación urbana.

Palabras Clave: Adolescentes - Felicidad - Marginación - Resiliencia.

1 Facultad de Comunicación Humana, Universidad Autónoma del Estado de Morelos, México

2 Facultad de Psicología, Universidad Autónoma del Estado de Morelos, México

3 Facultad de Psicología, Universidad Pablo de Olavide, España, elian_arte@yahoo.com 


\section{Abstract}

Urban marginalization in Mexico has greatly grown in the last years, affecting directly the youth. In this empirical study we analyzed its effect on the level of youth resilience and happiness, associated with structural factors. For this purpose the RESI-M questionnaire and the Happiness one-item Scale were applied to 1201 secondary and high schools Mexican students. Results showed that marginalization affects resilience dimensions differently. There were significant differences in resilience regarding gender, educational level and type of family. Statistical linear regression analysis confirmed the predictive value of some structural factors. We discuss how the marginal conditions affect the resilience level, however they could not predict it, as the individual can transform himself and his adverse reality. As for happiness it was not associated with the context conditions, except when the age and family are considered. The influence of the different types of family related to the marginalized context, as well as their role in the development of resilience to cope with the future challenges and avoid social maladjustment are analyzed. This study gives hints to deepen on the understanding of urban marginalized teenagers.

Keywords: Happiness - Marginalization - Resilience - Teenagers. 
Más de la mitad de la población juvenil de México vive con cierto grado de pobreza asociado a marginación (CEPAL, 2001; Díaz-Sánchez, 2006). En términos absolutos el mayor número de pobres y marginados viven en zonas urbanas como consecuencia del crecimiento poblacional aunado a la migración desmedida del campo a la ciudad y al aumento de la urbanización sin el incremento proporcional de empleos, servicios públicos, calidad de vida, etc. (CONAPO, 2009). La marginación urbana en México es un problema estructural (CONAPO, 2012), se define como una condición en la cual las personas no pueden acceder a los recursos y servicios que ofrece la urbe, ya sea por la falta de oportunidades o por ser segregados o discriminados, consecuencia en gran medida de la involución económica del país (Cueli \& Morales, 2002) y de un tejido social dañado que mantiene a ciertos sectores de la población al margen de las oportunidades y en condiciones de vida inaceptables (CEPAL, 2001; González et al., 2008). Además, la marginación se vuelve un ciclo transgeneracional (Cortes, 2006; Adler, 2003), en el cuál la población de bajos recursos se encuentra atrapada, desfavorecida por empleos informales asociados a bajos salarios y falta de seguridad social, que a su vez impide satisfacer las necesidades secundarias y en ocasiones, hasta las más básicas, como acceder a educación y servicios de salud de calidad al tener costos inalcanzables (Hernández-Pérez, 2010).

En la zona central de México, se encuentra el estado de Morelos, cuya ciudad capital es Cuernavaca, donde el $84 \%$ de la población vive en AGEB ${ }^{4}$ urbanas (INEGI, 2010), del cual el 70.9\% reportan condiciones de marginación ${ }^{5}$ (CONAPO, 2010); con un incremento del $4 \%$ en tan solo los últimos 5 años (CONAPO, 2005; 2012). De aquí la importancia de estudiar una muestra de estas localidades y especialmente una muestra de adolescentes, uno de los grupos más vulnerables a las condiciones precarias y marginales de vida. Vulnerables por la dinámica evolutiva de la edad, pero también, por las exigencias del entorno (CEPAL, 2001b). Es común que los adolescentes de hogares urbanos pobres y marginados, no cuenten con suficientes elementos protectores, por ejemplo, tienden a carecer de apoyo social y familiar, que probablemente los lleva a sentirse vulnerables (Martínez-Pizarro, 2000); se enfrentan a estratos sociales diferenciados; viven en constante contradicción y contrastación con sus pares; son cotidianamente excluidos o segregados de oportunidades (CEPAL, 2001b). Al

4 AGEB: Área Geo-Estadística Básica, medida básica de territorio utilizada por CONAPO.

5 Según el CENSO 2010, el 70.9\% de marginación contempla grado "medio" = 39.3\%; "alto" $=29.8 \%$; "muy alto" $=1.8 \%$. El otro $29 \%$ de la población viven en localidades con un grado de marginación "bajo" y "muy bajo". Datos obtenidos del "Índice de Marginación Urbana por localidad 2010". 
mismo tiempo, la marginación tiende a impactar negativamente en la salud física y psicológica, la dignidad, la autoestima y las oportunidades de vida de la persona (Prilleltensky, 2004); con una fuerte tendencia al desajuste social, afectando su bienestar (Ávila-Espada et al., 1996; Díaz-Morales \& Sánchez-López, 2001). Sin embargo, una gran parte de los adolescentes, como sujetos sociales activos (CEPAL, 2001), logran sobreponerse a todas estas vicisitudes y mantener niveles aceptables de felicidad, gracias a su capacidad de resiliencia (Janas, 2002; Cardozo $\&$ Alderete, 2009). Se han encontrado correlaciones positivas entre resiliencia y pobreza (Canvin et al., 2009; Seccombe, 2002; Garmezy, 1993).

Dado el crecimiento continuo de la marginación urbana en Cuernavaca y zonas aledañas, se vio la necesidad de analizar la capacidad de resiliencia y el nivel de felicidad de los adolescentes, temáticas fundamentales para un grupo etario considerado muy vulnerable al contexto adverso (CEPAL, 2001; Ávila-Espada et al., 1996; Díaz-Morales \& Sánchez-López, 2001). A nivel internacional, ha crecido el interés por factores como la resiliencia y la felicidad en poblaciones pobres y marginadas (Seccombe, 2002; González, 2004; Gómez et al., 2007; Szloboda, 2008; Canvin et al., 2009; Schimmel, 2009; Csikszentmihayli, 2010; Oshio \& Kobayashi, 2011; Palomar, Matus \& Victorio, 2012); mientras que las investigaciones locales, se han abocado a los factores negativos asociados a la marginación (Adler, 2003; Cortes, 2006; Cueli \& Morales, 2002; Díaz-Sánchez, 2006; Hernández-Pérez, 2010), por lo que se conoce muy poco al respecto de la resiliencia y felicidad de los adolescentes en estas regiones, a pesar de ser temas fundamentales para su bienestar social y psicológico.

La resiliencia es la capacidad del sujeto o grupo, para usar la experiencia derivada de las situaciones adversas, sobrellevarlas, recuperarse y/o proyectarse a futuro (Janas, 2002). Preserva la integridad en circunstancias difíciles; además ayuda a generar conductas adecuadas para la resolución de problemas y la sensación de control sobre la propia vida (Fiorentino, 2008). La resiliencia es multifactorial (Garmezy, 1993), sus fuentes se vinculan con la persona, sus características intrínsecas y sociales para enfrentar las dificultades que presenta el contexto. Se compone de factores como: fortaleza y confianza en sí mismo, competencia social, apoyo familiar, apoyo social y estructura (Palomar \& Gómez, 2010). A pesar de que hay discrepancias respecto de la importancia de cada factor (Cardozo \& Alderete, 2009) para algunos, el apoyo familiar y social son tan fundamentales como la fortaleza y confianza en uno mismo (Montero, 2004).

La resiliencia varía significativamente de acuerdo al sexo y la edad al estar ligada al desarrollo y crecimiento humano (Grotberg, 2003); es un factor en el cual se observan grandes diferencias individuales (Fiorentino, 2008), no se han visto diferencias significativas de acuerdo factores como la escolaridad, ocupación o 
nivel de ingresos (Palomar \& Gómez, 2010). Al mismo tiempo, estudios en otros países como Gran Bretaña y Estados Unidos de América muestran que existe una correlación positiva entre resiliencia y pobreza o inequidad (Canvin et al., 2009; Seccombe, 2002; Garmezy, 1993). En México se ha encontrado que en los grados más altos de marginación la capacidad de resiliencia se ve disminuida (González et al., 2008). Frente a esta diversidad de resultados, es imprescindible analizar los datos empíricos en base a la población de interés de este estudio, además, la resiliencia ha sido asociada a mayores niveles de bienestar o felicidad en situaciones de adversidad (Szloboda, 2008; Salgado, 2009; González, 2004).

La felicidad es el balance subjetivo de los afectos positivos y negativos que provoca una experiencia inmediata, tiene la característica de ser estable temporalmente y consistente en distintas situaciones (Benatuil, 2001). Para Diener (1994) la felicidad incluye además una dimensión cognitiva y se ha visto cómo el grado de calidad de vida favorable percibido, medida subjetiva (Schimmel, 2009), contribuye a mantener una concepción positiva de uno mismo (Csikszentmihalyi, 2010).

Existe un estereotipo social de que la felicidad aumenta en función de los ingresos (Sen, 2009), sin embargo, la mayoría de los investigadores coinciden en que la felicidad no está asociada a los ingresos económicos (Rojas, 2004; Layard, 2005; Oshio \& Kobayashi, 2011; Cuadra \& Florenzano, 2003). En países como México, donde más del 55\% de la población es pobre (PNUD, 2004), se observa que aunque hay una correlación positiva entre felicidad e ingresos, esta relación no es lineal (Rojas, 2004). Estudios tanto nacionales como internacionales, demuestran un porcentaje muy alto de pobres felices y de ricos muy infelices (Schimmel, 2009). Los niveles de felicidad relativamente altos entre la población pobre y marginada, implica tomar en cuenta la percepción subjetiva, sugiriendo una relación más compleja entre variables (Rojas, 2004), otra razón fundamental para analizar la percepción de los adolescentes marginados de Cuernavaca respecto a su nivel de felicidad.

Por las razones anteriores, se propuso lograr tres objetivos en este estudio: (1) Identificar el grado y el tipo de relación entre la marginación urbana, la felicidad y la resiliencia de los adolescentes. (2) Analizar las diferencias en la resiliencia y la felicidad de los jóvenes, al compararlos por grado de marginación, en función del género, edad, escolaridad, tipo de familia (parentalidad) y tipo de escuela a la que asisten. (3) Generar modelos explicativos de resiliencia y felicidad. 


\section{Método}

\section{Participantes}

Participaron de manera voluntaria 1158 adolescentes en base a un muestreo no probabilístico, 621 mujeres, 521 hombres y 16 no especificaron. Sus edades fluctuaron entre 12 y 19 años $(M=15.16 ; D E=1.71)$. Se agruparon en: adolescencia temprana de 12 a 14 años (35.4\%, $n=410)$; adolescencia media de 15 a 17 años $(54.7 \%, \mathrm{n}=634)$; adolescencia tardía de 18 a 19 años $(8.2 \%, n=95)$; y $1.6 \%(n=19)$ no especificaron su edad. Del total $47 \%(n=544)$ estudian en la secundaria, $51.4 \%(n=595)$ estudian en el bachillerato y $1.6 \%(n=19)$ no especificaron. El $22.6 \%$ estudian en escuelas privadas y el $77.4 \%$ en escuelas públicas. El $19 \%$ de los participantes trabajan en paralelo a sus estudios. El 76.3\% proviene de familias biparentales; $16.1 \%$ proviene de familias monoparentales ( $84.8 \%$ solo con la madre y $15.2 \%$ vive solo con el padre) y $7.6 \%$ no cuentan con figuras parentales. En relación al grado de marginación en que viven, el $29 \%(n=336)$ habita en AGEB con un grado "bajo" de marginación; el 40.6\% $(n=$ $470)$ en un grado "medio"; el 26.7\% $(n=309)$ en grado "alto" de marginación, y el $3.7 \%(n=43)$ no especifican localidad de vivienda. Se excluyeron los jóvenes de más de 19 años y los que viven en zonas de muy baja marginación.

\section{Instrumentos}

Se utilizaron dos instrumentos de autoaplicación: RESI-M (Palomar \& Gómez, 2010) y la Escala de Felicidad (Abdel-Khalek, 2006), junto con una encuesta sociodemográfica para conocer género (hombre y mujer), edad (tres grupos: 12-14 años, 15-17 años, 18-21 años), escolaridad (secundaria y bachillerato), tipo de escuela (privado o público), tipo de familia-monoparental (vive con solo padre o solo madre), biparental (vive con ambos padres) y sin figuras paternas biológicas (vive con otros adultos)-. La Escala de Resiliencia RESI-M desarrollada para poblaciones mexicanas (Palomar \& Gómez, 2010), se basa en Connor-Davidson Resilience Scale (CD-RISC) y Resilience Scale for Adults (RSA). Cuenta con 43 reactivos; su nivel de confiabilidad fue de $\alpha=.959$; el análisis factorial por rotación Varimax arrojó cinco dimensiones que explican $54.69 \%$ de la varianza: fortaleza y confianza en símismo, $\alpha=.931$; competencia social, $\alpha=.865$; apoyo familiar, $\alpha=.892$; apoyo social, $\alpha=.891$; estructura vital, $\alpha$ $=.797$. La Escala de Felicidad de un ítem “ ¿Te sientes feliz en general?”, donde 0 es nada feliz y 10 muy feliz (Abdel-Khalek, 2006), tiene una estabilidad temporal de .86. Para determinar su validez fue correlacionada con el Inventario de Felicidad de Oxford (OHI) y la Escala de satisfacción con la vida de Diener, Emmons, Larsen y Griffin y resultó ser altamente significativa (Abdel-Khalek, 2006). 


\section{Procedimiento}

Con el permiso de las autoridades de los colegios, se llevó a cabo la autoaplicación voluntaria de los cuestionarios, como parte de un macro-estudio del equipo de investigación UAEM-UPO, que incluyó una batería de 22 instrumentos. Se realizaron análisis estadísticos descriptivos e inferenciales con el programa estadístico SPSS (versión 19).

Para conocer el nivel de marginación de los jóvenes, se solicitó la base de datos del Consejo Nacional de Población (CONAPO), quien analiza factores socio-económicos, condiciones de la vivienda y acceso a los servicios públicos y educación para clasificar las localidades en cinco niveles de marginación desde muy alto hasta muy bajo. A los efectos de la presente investigación, dado que la población estudiada estaba escolarizada, sólo se consideraron adolescentes en condiciones de alto, media y bajo nivel de marginación, se excluyó el extremo de muy bajo nivel de marginación.

\section{Resultados}

Se realizaron análisis de correlación de Pearson entre el grado de marginación, la resiliencia, sus subindices y la felicidad (ver Tabla 1). La relación entre marginación y resiliencia resultó negativa y significativa, no obstante el coeficiente de correlación fue muy bajo. En el caso de los subíndices de resiliencia, se obtuvieron correlaciones negativas y significativas entre el grado de marginación y los factores fortaleza y confianza en sí mismo, apoyo familiar y apoyo social, mientras que no hubo correlación entre marginación y los factores competencia social y estructura vital de la Escala de Resiliencia, tampoco hubo correlación entre marginación y felicidad. Esto último sugiere que la competencia social y estructura vital percibida por los adolescentes, al igual que su nivel de felicidad, son independientes del grado de marginación en el que viven. 
Gómez-Azcarate, Vera , Ávila , Musitu, Vega \& Dorantes I Psicodebate, 14(1) I 45-68.

\section{Tabla 1.}

Matriz de coeficientes de correlación, medias y desviaciones estándar.

\begin{tabular}{|c|c|c|c|c|c|c|c|c|}
\hline & 1 & 2 & 3 & 4 & 5 & 6 & 7 & 8 \\
\hline 1. Felicidad & 1 & & & & & & & \\
\hline 2. Resiliencia & $.166^{\star \star}$ & 1 & & & & & & \\
\hline 3. Marginación & -.034 & $-.073^{\star}$ & 1 & & & & & \\
\hline 4. Fortaleza & $.117^{\star *}$ & $.933^{\star \star}$ & $-.070^{\star}$ & 1 & & & & \\
\hline 5. Comp. social & $.090^{\star *}$ & $.800^{\star *}$ & -.044 & $.678^{* *}$ & 1 & & & \\
\hline 6. Apoyo familiar & $.131^{\star \star}$ & $.727^{\star \star}$ & $-.070^{\star}$ & $.591^{\star \star}$ & $.493^{\star *}$ & 1 & & \\
\hline 7. Apoyo social & $.217^{\star *}$ & $.772^{\star *}$ & $-.069^{*}$ & $.625^{\star \star}$ & $.493^{\star *}$ & $.541^{\star *}$ & 1 & \\
\hline 8. Estructura vital & $.174^{\star \star}$ & $.725^{\star \star}$ & -.035 & $.577^{\star \star}$ & $.505^{\star \star}$ & $.507^{\star \star}$ & $.537^{\star \star}$ & 1 \\
\hline$M$ & 83.73 & 71.13 & 2 & 71.75 & 68.75 & 79.66 & 70.85 & 64.36 \\
\hline$D E$ & 20.51 & 16.74 & & 18.17 & 20.46 & 21.19 & 23.89 & 21.89 \\
\hline \multicolumn{9}{|c|}{$\begin{array}{l}\text { Nota: M y DE son valores estandarizados. Para grado de marginación, se presenta la Mo } \\
(2=\text { grado medio de marginación). }\end{array}$} \\
\hline
\end{tabular}

Para responder al segundo objetivo, se realizaron análisis de comparación de medias independientes con Pruebas $t$ de Student, de la resiliencia y la felicidad de los adolescentes, en función del grado de marginación urbana de su localidad y sus características estructurales como género, edad, escolaridad, tipo de familia y tipo de escuela (ver Tabla 2). Los resultados mostraron que no existen diferencias significativas en la comparación de medias de la resiliencia y la felicidad de los adolescentes, en función de las condiciones de marginación. Resaltan los altos puntajes promedio de resiliencia $(M=71.13, D E=16.74)$ y felicidad $(M=83.73$, $D E=20.51)$ de todos los participantes $(n=1158)$. 


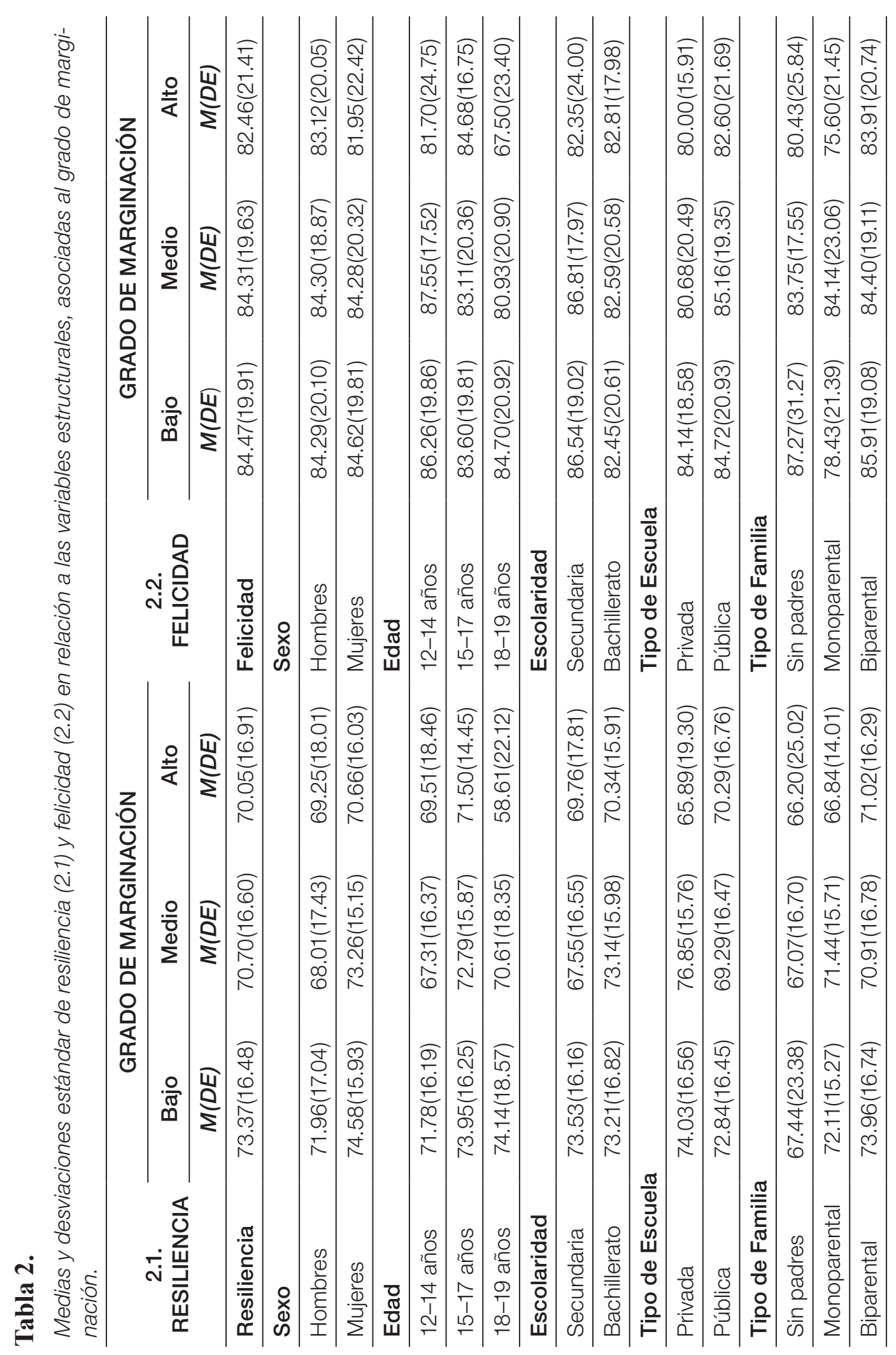


En cuanto a las características estructurales de los participantes, al comparar la resiliencia entre los tres grupos de marginación, se encontraron diferencias significativas entre la resiliencia de los jóvenes el nivel bajo y medio $(t=2.56, p$ $<.05)$ y entre el nivel bajo y alto de marginación $(t=2.52, p<.05)$. No habiendo diferencias en el nivel de felicidad. Si lo comparamos por género, los resultados mostraron una tendencia generalizada de las mujeres participantes a tener una mayor capacidad de resiliencia que los hombres. Sin embargo, sólo se observaron diferencias estadísticamente significativas en condiciones de marginación media $(t=-3.49, p<.001)$. Respecto a la edad de los participantes en función del nivel de marginación, los resultados mostraron que cuando viven en zonas de media marginación, la resiliencia es significativamente mayor en el grupo de 15 a 17 años $(t=-3.35, p<.001)$, comparado con el de 12 a 14 años. En marginación alta, hubo diferencias entre los jóvenes de 12 a 14 años y los de 18 a 19 años $(t=2.01, p<.005)$; también es mayor en el de 15 a 17 años que de los 18 a 21 años $(t=2.94, p<.005)$. Mientras que en las condiciones de marginación baja no hubo diferencias en el nivel de resiliencia respecto al grupo de edad. En cuanto a la escolaridad en función del grado de marginación, sólo hubo diferencias significativas en las condiciones de marginación media $(t=-3.66, p<.001)$, siendo los de bachillerato los que mostraron mayor capacidad de resiliencia. En las condiciones de alta y baja marginación, la escolaridad no afecta el nivel de resiliencia. Los resultados respecto a la resiliencia en función del tipo de escuela a la que asisten -pública o privada-asociada al grado de marginación, mostraron diferencias significativas sólo cuando los adolescentes viven en condiciones de marginación media $(t=-3.96, p<.001)$, siendo más alto el nivel de resiliencia de los adolescentes que asisten a escuelas privadas, pero que viven en condiciones de marginación media. Estas diferencias no fueron significativas en las comparaciones de los otros grupos según sus condiciones de marginación.

Al considerar si el tipo de familia a la que pertenecen, asociado al grado de marginación, manifestaban diferencias en la resiliencia de los adolescentes y se encontró que en las zonas de baja marginación, el no tener padres presentes -sin figuras paternas- comparado con tener al menos uno de los padres -familia monoparental- presentes provocó un nivel de resiliencia significativamente menor en los adolescentes $(t=2.16, p<.05)$. Al tener a ambos padres en casa -familia biparental-, hubo una diferencia significativa mayor, que no teniendo a ninguno -sin figuras paternas $-(t=-2.73, \mathrm{p}<.05)$. Respecto a las familias monoparentales (16.4\% de los casos), se comparó la resiliencia en función de la presencia de la madre vs. la del padre en el hogar independientemente del grado de marginación. Los resultados mostraron que la presencia de la madre se asocia significativamente con niveles más altos de resiliencia $(\mathrm{t}=3.29, \mathrm{p}<.001)$.

Además se realizaron comparaciones para cada subíndice por separado en 
función de las condiciones de marginación, los resultados mostraron diferencias significativas entre baja y media marginación en F1: fortaleza y confianza en sí mismo $(t=2.25, p<.05) ; F 3$ : apoyo familiar $(t=2.82, p<.05) ; F 4$ : apoyo social $(t=2.41, p<.05)$. Entre baja y alta marginación en $F 1(t=2.45, p<.05) ; F 3$ : apoyo familiar $(t=2.47, p<.05) ; F 4$ : apoyo social $(\mathrm{t}=2.27, \mathrm{p}<.05)$. Entre media $y$ alta marginación no hubo diferencias significativas.

En cuanto a la variable felicidad, se utilizó el mismo procedimiento para comparar las medias de muestras independientes con pruebas $t$ de Student, segmentando la muestra por grado de marginación (ver Tabla 2, sección 2.2. Felicidad). Los resultados mostraron que no hubo diferencias significativas entre la felicidad de hombres y mujeres, asociado a la marginación en la que viven. En relación al grupo de edad, se observó una tendencia a que en el grado de marginación más alto, la felicidad se redujera en función de la edad, sin embargo esta diferencia no fue significativa. Sólo en condiciones de marginación media, se observaron diferencias significativas al comparar la felicidad de los adolescentes de 12 a 14 años y los de 15 a 17 años $(t=2.17, p<.05)$ y entre los de 12 a 14 años y 18 a 19 años $(\mathrm{t}=2.06, \mathrm{p}<$ $.05)$, en ambos casos, fueron los más jóvenes quienes reportaron ser más felices. Por otro lado, también hubo diferencia en las medias de felicidad de los adolescentes en relación a la escolaridad (secundaria o bachillerato), en grado de marginación media $(t=2.23, p<.05)$ los de secundaria dicen ser más felices. No hubo diferencias en la felicidad en función del tipo de escuela (privada o pública). En cuanto al tipo de familia, hubo pocas diferencias en el nivel de felicidad, sólo en dos casos: a) Cuando viven con ambos padres -familias biparentales- en condiciones de baja marginación, la puntuación media de felicidad fue significativamente más alta que cuando viven en familias monoparentales $(t=-2.77, p<.01)$. b) Igualmente sucede cuando viven en condiciones de alta marginación $(t=-2.34, p<.05)$, no así en condiciones de marginación media. Finalmente, no fueron significativas las puntuaciones de felicidad, en las comparaciones vivir sólo con madre o padre, como jefe de familia monoparental.

Para responder al tercer objetivo se realizaron análisis estadísticos de Regresión Lineal Múltiple a continuación se describen.

\section{Modelos explicativos del nivel de resiliencia}

Para indagar si las variables estructurales explicaban el nivel de resiliencia, se construyeron dos modelos, en el Modelo 1 (M1) se consideraron las variables estructurales de interés, en el Modelo 2 (M2) se agregó el nivel de felicidad. Los análisis de regresión, permitieron corroborar los resultados anteriores y diferenciar el peso explicativo de cada factor estructural (ver Tabla 3 de los coeficientes 
estimados). Los resultados mostraron que la capacidad explicativa de ambos modelos, es débil pero significativa: $M 1\left(\mathrm{R}^{2}=.02, p<.002\right), M 2\left(\mathrm{R}^{2}=.03, p<.000\right)$. En el primer modelo sólo el sexo, el tipo de familia, y el tipo de escuela, explicaron el nivel de resiliencia de los adolescentes. Mientras que la edad, la escolaridad y el grado de marginación, no fueron predictores de la resiliencia. El segundo modelo mostró que el nivel de felicidad junto con el sexo, tipo de familia y tipo de escuela, fueron buenos predictores del nivel de resiliencia de los adolescentes.

Tabla 3.

Modelo explicativo del nivel de resiliencia.

\begin{tabular}{lcc}
\hline & Modelo 1 & Modelo 2 \\
\hline Variables & $\boldsymbol{B}(\mathrm{EE})$ & $\boldsymbol{B}(\mathrm{EE})$ \\
\hline Constante & $74.03(6.74)^{\star \star \star}$ & $61.74(7.04)^{\star \star \star}$ \\
\hline Sexo & $3.01(1.01)^{\star \star}$ & $3.01(1.00)^{\star}$ \\
\hline Edad & $-.79(.49)$ & $-.71(.49)$ \\
\hline Tipo de Familia & $2.07(.87)^{\star}$ & $1.92(.87)^{\star}$ \\
\hline Escolaridad & $3.44(1.67)^{\star}$ & $3.55(1.65)^{\star}$ \\
\hline Tipo de Escuela & $-2.67(1.33)^{\star}$ & $-2.86(1.31)^{\star}$ \\
\hline Marginación & $-1.06(.69)$ & $-.88(.68)$ \\
\hline Felicidad & & $.13(.02)^{\star \star \star}$ \\
\hline $\mathrm{R}^{2}$ & .028 & .055 \\
\hline $\mathrm{F}$ & $5.11^{\star \star \star}$ & $8.64^{\star \star \star}$ \\
\hline${ }^{* \star \star} p<.001,{ }^{\star \star} p<.01,{ }^{\star} p<.05, B=$ coeficientes, EE = error estándar \\
\hline
\end{tabular}

Posteriormente, para explicar el nivel de resiliencia diferenciando el grado de marginación, se segmentó la muestra por grado de marginación obteniendo modelos de regresión separados (ver Tabla 4). Se observó que la capacidad explicativa de los modelos en función del grado de marginación, resultó muy débil aunque todos fueron significativos. En el caso del modelo para el grado medio, las variables estructurales significativas fueron todas excepto tipo de familia. Mientras que en el caso de bajo y alto grado de marginación, sólo felicidad fue significativa dentro de los modelos. 
Tabla 4.

Modelos de explicación del nivel de resiliencia según grado de marginación.

\begin{tabular}{|c|c|c|c|}
\hline \multirow{3}{*}{ Factores } & \multicolumn{3}{|c|}{ GRADO DE MARGINACIÓN } \\
\hline & Bajo & Medio & Alto \\
\hline & $B(E E)$ & $B(E E)$ & $B(E E)$ \\
\hline Constante & $52.2(6.75)^{\star \star \star}$ & $48.49(5.83)^{\star \star \star}$ & $48.97(7.15)^{\star \star \star}$ \\
\hline Sexo & $2.68(1.82)$ & $4.39(1.51)^{\star \star}$ & $1.02(1.93)$ \\
\hline Tipo de Familia & $1.65(1.78)$ & $1.44(1.26)$ & $2.88(1.59)$ \\
\hline Escolaridad & $-.28(1.98)$ & $4.36(1.61)^{\star \star}$ & $.25(1.94)$ \\
\hline Tipo de Escuela & $-1.29(1.99)$ & $-5.74(1.98)^{\star \star}$ & $6.53(4.19)$ \\
\hline Felicidad & $.18(.05)^{\star \star \star}$ & $-.13(.04)^{\star \star \star}$ & $.10(.45)^{\star}$ \\
\hline $\mathrm{R}^{2}$ & .06 & .08 & .04 \\
\hline$F$ & $4.04^{\star \star \star}$ & $8.46^{\star \star \star}$ & $2.55^{\star}$ \\
\hline
\end{tabular}

\section{Modelos explicativos del nivel de felicidad}

Se generaron dos modelos explicativos a partir de regresiones lineales, para conocer si las variables estructurales predecían el nivel de felicidad, sin embargo, los resultados mostraron que los modelos no fueron significativos, por lo cual no se reportaron. La única variable explicativa del nivel de felicidad observada en ambos modelos, aunque con un peso muy bajo, fue el tipo de familia $(B=.05, E E=.02, p$ $<.05)$. Sin embargo, se realizaron otros análisis de Regresión Lineal para predecir la felicidad en base a la resiliencia y sus componentes (ver Tabla 5). Se generaron dos modelos, en uno donde sólo se introdujo la resiliencia y en otro se introdujo además de la resiliencia, sus dimensiones por separado. Ambos resultaron con una capacidad predictiva baja aunque significativa. En el segundo modelo, sólo apoyo social y estructura vital fueron predictores significativos de la felicidad. Con estos resultados, se corroboró que el nivel de felicidad percibido por los adolescentes explica su capacidad de resiliencia, al mismo tiempo la resiliencia, a través de sus subíndices de apoyo social y estructura vital, explica la felicidad. 
Tabla 5.

Modelos explicativos del nivel de felicidad.

\begin{tabular}{lcc}
\hline \multirow{2}{*}{ Factores } & Modelo 1 & Modelo 2 \\
\cline { 2 - 3 } & $\boldsymbol{B}(\mathrm{EE})$ & $\boldsymbol{B}(\mathrm{EE})$ \\
\hline Constante & $70.48(2.80)^{\star \star \star}$ & $70.48(2.78)^{\star \star \star}$ \\
\hline Resiliencia & $.09(.03)^{\star \star}$ & $-.07(.05)^{\star}$ \\
\hline R1 (fortaleza) & $-.07(.05)$ \\
\hline R2 (comp. Social) & $-.03(.04)$ \\
\hline R3 (apoyo familiar) & $.02(.04)$ \\
\hline R4 (apoyo social) & $.17(.03)^{\star \star \star}$ \\
\hline R5 (estructura) & $-.09(.04)^{\star \star}$ \\
\hline$R^{2}$ & .010 & .055 \\
\hline$F$ & $11.15^{\star \star \star}$ & $12.53^{\star \star \star}$ \\
\hline${ }^{* \star *} p<.000,{ }^{* \star} p<.001,{ }^{*} p<.05 B=$ coeficiente, $E E=$ error estándar. \\
\hline
\end{tabular}

\section{Discusión y Conclusiones}

En base a los resultados, se encontró una tendencia en la que conforme se incrementa el grado de marginación urbana disminuye la capacidad de los adolescentes a resistir y recuperarse de la adversidad, aunque fue débil la relación negativa entre marginación y resiliencia. Los resultados coinciden con las descripciones de Cortes (2006) y Adler (2003), pero entran en contradicción con los resultados empíricos de Canvin y colaboradores (2009) y Seccombe (2002), probablemente, debido a que los contextos socioeconómicos marcan una diferencia diametral en la construcción de la resiliencia de los sujetos (Prilleltensky, 2004; Ávila-Espada et al., 1996; Díaz-Morales \& Sánchez-López, 2001). En este sentido, es fundamental considerar que si la posibilidad del adolescente mexicano para utilizar las experiencias provenientes de las situaciones adversas se ve reducida por la marginación misma les será difícil sobrellevarlas, recuperarse o proyectarse a futuro. Esto limita enormemente a los jóvenes más marginados y los mantiene en un estado de indefensión y de fragilidad social, como lo han planteado investigadores de CEPAL (2001).

En el caso de la felicidad, ésta no se asoció directamente al grado de marginación, en general se observaron niveles elevados de felicidad en todos los participantes, similares a los resultados de la encuesta piloto del INEGI (2012) para la población de 17 a 80 años en México. Lo anterior coincide con los planteamientos de otros investigadores de que la marginación y la pobreza no afectan el nivel de felicidad (Rojas, 2004; Cuadra \& Florenzano, 2003), similar al planteamiento teórico y las observaciones de que la felicidad no está asociada a los ingresos económicos (Sen, 
2009; Layard, 2005; Oshio \& Kobayashi, 2011). En condiciones de marginación media, la felicidad se ve comprometida al comparar grupos de edad y escolaridad, mientras que en los grupos de alta y baja marginación varía en función del tipo de familia. Esto implica que algunos factores externos afectan la felicidad de los jóvenes mexicanos pero en mucho menor grado de lo que afectan su resiliencia. Es decir, que la resiliencia se ve más comprometida por el contexto que por la felicidad. Lo cual nos lleva a suponer que los adolescentes mexicanos, aunque no tengan una gran capacidad para recuperarse y/o hacer frente a la adversidad de su cotidianeidad, logran mantener niveles aceptables de bienestar, independientemente de las condiciones del contexto socioeconómico en la que vivan. Además, la felicidad como equilibrio del bienestar subjetivo se da normalmente, por lo que no se ve fuertemente comprometida por el contexto, excepto si las condiciones son realmente extremas, como no tener qué comer, etc., como se ha planteado en estudios anteriores, al ser un constructo no asociado directamente al grado de pobreza (Layard, 2005), sino al referente conceptual individual (Rojas, 2007). Vivir en hogares urbanos pobres, con menos oportunidades de salir adelante, enfrentándose a estratos sociales diferenciados, en constante contradicción con sus pares en mejores condiciones, en cierta medida, tiene un impacto en la vida de los jóvenes, pero este no es directamente en su percepción de felicidad, sino sólo en su capacidad de resiliencia.

Por otro lado, en cuanto a la relación entre felicidad y resiliencia, se corroboró que es positiva, por lo que a mayor capacidad de resiliencia mayor nivel de felicidad, en concordancia con investigaciones anteriores sobre la asociación de ambas variables (Szloboda, 2008; Salgado, 2009). A través de los análisis de regresión lineal se observó que la felicidad percibida por los adolescentes tiene más capacidad explicativa que la propia resiliencia; aunque la resiliencia a través de sus subíndices de apoyo social y estructura vital, pueden explicar el nivel de felicidad. Esto supone no sólo una asociación bidireccional entre ambas variables, sino la relación causal en la cual se puede predecir más fácilmente la resiliencia si se conoce el nivel de felicidad de los adolescentes. Estos hallazgos, aunque alentadores en términos absolutos por lo que implican en cuanto a la posibilidad real de ser felices aún a costa de la adversidad, deben ser vistos con cautela y profundizar en su análisis comenzando por considerar las características individuales y sociodemográficas asociadas al grado de marginación que pueden explicar el nivel de felicidad y resiliencia. Además, sería prudente considerar que los resultados parten de la percepción subjetiva e individual que los adolescentes tienen.

Al profundizar en el estudio de la resiliencia a través de diferenciar sus componentes, se observó que al aumentar el grado de marginación disminuyeron de manera significativa la fortaleza y confianza en sí mismo, el apoyo familiar y el apoyo social, mientras que la competencia social y la estructura vital, no se 
vieron afectadas por las condiciones de vida marginal. Además, la percepción del apoyo familiar y de su estructura vital resultaron ser los componentes más débiles cuando se compararon adolescentes en grados altos de marginación urbana, quienes en general mostraron menor capacidad para enfrentarse a su realidad. Se ha planteado que los grupos más marginados tienden a contar con menos elementos protectores y que pudiera haber una tendencia menor a una falta de apoyo social y familiar que conllevara al individuo a sentirse vulnerable como lo ha planteado Martínez-Pizarro (2000), lo cual está relacionado con un tejido social dañado (CEPAL, 2001; González et al., 2008) que perpetúa las condiciones de vida de generación en generación (Cortes, 2006; Adler, 2003) a las cuales, probablemente, los jóvenes se habitúan y normalizan (Montero, 2004).

Posteriormente, al ahondar en el estudio de la resiliencia en función de las variables estructurales de interés, se observó la mayor variabilidad en la muestra en condiciones de marginación media donde influye el género, la escolaridad, el tipo de escuela al que asisten. Para los adolescentes viviendo en condiciones de alta marginación, las diferencias se asociaron a la edad y, en el grado de marginación bajo, se asociaron a contar o no con ambos padres.

En estos análisis, al comparar la resiliencia en función del género y el grado de marginación, se observó una tendencia generalizada de las mujeres participantes a tener una mayor capacidad de resiliencia que los hombres, sin embargo, solo fue significativa en condiciones de marginación media. Pocos estudios han explorado la asociación entre género y marginación para explicar la capacidad de resiliencia, pero es fundamental entender las diferencias en la percepción que los hombres y mujeres tienen de su fortaleza y confianza en sí mismos, de su competencia social, así como del apoyo familiar y social que reciben, especialmente al vivir en contextos marginados, con niveles altos de pobreza, carencia de servicios y oportunidades, pero en el cual las mujeres se perciben a sí mismas como más capaces de sobrellevar las condiciones de vida en las que están. En este sentido, independientemente de las condiciones de vida marginal, existen numerosos estudios que han reportado diferencias de género en el nivel de resiliencia, la mayoría han afirmado que las mujeres tienden a mostrar niveles más altos de resiliencia que los hombres (Palomar \& Gómez, 2010; Romer et al. 2011; Hystad, 2012; Wasonga et al., 2003), pero no todos los estudios coinciden (Stratta et al., 2013; González, Valdez, \& Zavala, 2008), lo cual implica que las diferencias probablemente se relacionan con otros elementos del contexto social, de la educación, o del sujeto, como la percepción y expectativas sobre uno mismo, etc.

Al mismo tiempo, al analizar el efecto de la edad en los niveles de resiliencia, se encontró que los participantes en la etapa de adolescencia media, fueron quienes reportaron una mejor capacidad de resiliencia a pesar de las condiciones 
adversas en las que viven. Mientras que los adolescentes más jóvenes, mostraron resultados en forma de $\mathrm{U}$ en función del grado de marginación, es decir, que los menos resilientes fueron los que se encontraron en el grado medio de marginación. En general, los participantes en la etapa de adolescencia tardía, mostraron menor capacidad de resistencia a las condiciones en los tres grados de marginación en los que se les agrupó según la colonia en la que viven.

Pareciera que con la edad, los adolescentes que viven en condiciones más adversas van perdiendo la capacidad de resistir, tal vez porque confrontan sus capacidades con las oportunidades reales de integración al mundo adulto formal (CEPAL, 2001). Es de considerarse que los jóvenes en adolescencia tardía, están rezagados escolarmente, pues en México un joven mayor de 18 años, debería ya estar cursando sus estudios superiores y sin embargo, la muestra reportó que una décima parte del total de adolescentes encuestados, tiene una edad mayor a la esperada para el grado que cursa, lo cual sugiere que, probablemente, los adolescentes desfasados no tengan tantos recursos personales y sociales no solo para salir adelante en sus estudios, sino para confrontar las dificultades de la vida.

En cuanto a la escolaridad y al tipo de escuela en función del grado de marginación, se observó que sólo influyen en las condiciones de marginación media. Los adolescentes de bachillerato mostraron mayor resiliencia que los de secundaria; al mismo tiempo, los que asisten a escuelas privadas cuentan con mayores niveles de resiliencia que los de escuelas públicas. Es decir que la escolaridad y el tipo de escuela no son elementos que impacten al joven cuando está en condiciones extremas - de alta o baja marginación-, pero se vuelven factores que afectan la capacidad de sobrellevar las dificultades, en las condiciones donde hay mayor variabilidad social, pues se convive tanto con personas con mayor y menor nivel de marginación. Además, el hecho de que sean los de bachilleratos privados los que tengan mayor resiliencia se relaciona con la madurez y el apoyo con el que cuenta dicha población.

Se analizó el nivel de resistencia con base al tipo de familia y en función de la marginación, y se observó una tendencia generalizada a que sea menor en los adolescentes viviendo sin figuras paternas biológicas con otros adultos que en los que viven en familias monoparentales o biparentales, sin embargo, fue en las zonas de baja marginación donde se observó mayor influencia el hecho no tener padres sobre el nivel de resiliencia. Se resalta que el tipo de familia fue la única variables estructural estudiada que afectó el nivel de felicidad reportado por los adolescentes de la muestra en función de sus condiciones de vida.

Es importante aclarar que los tipos de familia que se exploraron en este estudio, se diferenciaron en función de la presencia de los padres. Es notorio cómo se corroboró que las familias monoparentales o carentes de figuras parentales son 
en ocasiones entornos familiares adversos, no solo por las carencias económicas que esto implica, sino por las consecuencias en la capacidad de sobrellevar la adversidad y de mantener niveles aceptables de bienestar que ocasiona en los hijos adolescentes. Estas familias, al no ofertar suficiente apoyo familiar, orillan a los jóvenes a construir su propia fortaleza interna, a desarrollar competencias de socialización y a valerse por sí mismos. Por lo tanto, como se ha manifestado constantemente, las características del entorno familiar constituyen elementos determinantes en la adquisición y consolidación de capacidades que pueden llegar a definir la integración o marginación del adolescente en la transición hacia la vida adulta (CEPAL, 2001). Esto se corrobora en la regresión lineal múltiple donde el tipo de familia predice el nivel de resiliencia.

Históricamente, las familias monoparentales tienden a ser constituidas por la madre como jefe de familia (Katzman, 1992) quien es considerada como la más interesada en mantener los lazos familiares y quien ayuda a adquirir gran parte de las competencias sociales. Dicho planteamiento se confirmó con los hallazgos del presente estudio, donde la presencia de la madre fue más importante para el adolescente, alcanzando niveles significativamente más altos de resiliencia. No sucedió lo mismo en el caso de la felicidad de los jóvenes, puesto que a pesar de vivir en familias monoparentales, el nivel de felicidad fue similar al contar con ambos padres.

La familia es fundamental para el desarrollo del adolescente al ser un espacio de preparación en el cual aprende a afrontar retos, asumir responsabilidades, compromisos que lo orientan hacia una adultez plena. Es un lugar donde se puede encontrar empuje motivacional para afrontar el futuro (Musitu, Estévez, Martínez, \& Jiménez, 1993). Es decir, que quien no cuenta con figuras paternas probablemente tienda al desajuste social, en ocasiones, otros miembros de la comunidad llegan a cubrir estas carencias afectivas y sustituyen la necesidad parental, en este sentido, las familias pobres históricamente han subsistido gracias al apoyo de la familia extensa, de abuelos, tíos, etc. que ocupan dichos roles, no sucede lo mismo en las familias de mayor nivel socioeconómico como se ve en el presente estudio, donde la resiliencia y felicidad de los adolescentes menos marginados se ve afectada por el tipo de familia en la que viven.

Se vaticinaba que las variables estructurales influirían en la resiliencia (Palomar \& Gómez, 2010), sin embargo, pocas investigaciones han obtenido resultados de cómo la resiliencia de los adolescentes puede fluctuar no solo en relación a las variables estructurales per se, sino en función del grado de marginación. Las condiciones de vida marginales aunque afectan la capacidad de resiliencia de los adolescentes, no la predicen, pues el individuo puede transformarse a sí mismo y a su realidad adversa. Además, se confirmó que la felicidad no se explica en función de las condiciones del contexto, sino en función de lo que representa o 
significa para cada quien; en este sentido Rojas (2007) planteó que existe una heterogeneidad de referentes conceptuales más importantes que las condiciones de vida en sí mismas.

A modo de reflexión, se retoman las palabras del Consejo Nacional de Población (2002) que plantean que la marginación al ser un problema estructural, ofrece pocas oportunidades para adolescentes y sus familias, requiriendo además de niveles aceptables de fortaleza interna y confianza en uno mismo, apoyo social y familias, es decir, del trabajo activo de todos los agentes involucrados para aumentar los niveles de resiliencia de los adolescentes. Como colofón es necesario retomar el planteamiento de Seccombe (2002), quien considera esencial trabajar a nivel de la prevención y del desarrollo de políticas públicas que permitan a las futuras generaciones de jóvenes no tener que vivir y resistir los embates del problema psicosocial estructural llamado marginación urbana. En este sentido, el reto está no sólo en fortalecer las dimensiones de la capacidad de resiliencia, sino en transformar la realidad social.

\section{Limitaciones del estudio y próximos desafíos}

Para futuras investigaciones, se pretende ahondar sobre las representaciones sociales de la felicidad, cuando el grado de marginación no juega ningún papel, en especial en una población que históricamente ha vivido marginada ¿será que se ha habituado a esta condición? Habría que indagar más sobre los procesos de habituación y normalización (Montero, 2004) de las condiciones de marginación para entender si estos procesos influyen en la indiferencia al contexto al momento de valorar el grado de felicidad que experimenta el participante y que reporta en una escala gradual con diez opciones de respuesta.

Los datos sobre la felicidad de los adolescentes puede relacionarse a diversos factores, desde los más positivos, como la actitud ante la vida y la trascendencia humana que abstrae al sujeto de las necesidades materiales para ser feliz a pesar de las dificultades y de privaciones, hasta los factores más negativos como la indiferencia, negación y/o normalización de su contexto que no afecta su nivel de felicidad; no obstante, se puede caer en el error de mantener las inequidades al considerar que estas no afectan el bienestar de la población más necesitada (Friedli, 2012). 


\section{Referencias}

Abdel-Khalek, A. (2006). Measuring Happiness with a single-item scale. Social Behavior and Personality, 34(2), 139-150.

Adler, L. (2003). Cómo sobreviven los marginados. México: Siglo XXI Editores.

Ávila-Espada, A., Jiménez-Gómez, F. \& González-Martínez, M. (1996). Aproximación psicométrica a los patrones de personalidad y estilos de afrontamiento del estrés en la adolescencia: perspectivas conceptuales y técnicas de evaluación. En M. Casullo (Ed.), Evaluación psicológica en el campo de la salud. Barcelona: Paidós-Ibérica.

Benatuil, D. (2001). El bienestar psicológico en adolescentes desde una perspectiva cualitativa. Psicodebate. Psicología, Cultura y Sociedad, 3, 43-58.

Canvin, K., Marttila, A., Burstrom, B. \& Whitehead, M. (2009). Tales of the unexpected? Hidden resilience in poor households in Britain. Social Science \& Medicine, 69(2), 238-245.

Cardozo, G. \& Alderete, A. (2009). Adolescentes en riesgo psicosocial y resiliencia. Psicología desde el Caribe, 23, 148-182.

CEPAL (2001a). Informe de la reunión de expertos sobre jóvenes marginados en México, El Salvador, Nicaragua y Panamá. Publicaciones Electrónicas de la Comisión Económica Para América-Latina y el Caribe, Naciones Unidas.

CEPAL (2001b). Marginados en México, El Salvador, Nicaragua y Panamá. Publicaciones Electrónicas de la Comisión Económica Para América-Latina y el Caribe, Naciones Unidas.

CONAPO (2002). Concepto y dimensiones de la marginación a nivel localidad. México D.F.

CONAPO (2009). Índice de Marginación Urbana 2005, Consejo Nacional de Población, México D.F.

CONAPO (2012). Índice de Marginación Urbana 2010, Consejo Nacional de Población, México D.F.

Cortes, F. (2006). Consideraciones sobre la marginación, la marginalidad, la marginalidad económica y la exclusión social. Papeles de población, 47, 71-84. 
Csikszentmihalyi, M. (2010). Fluir (Flow): Una psicología de la felicidad. Barcelona: Editorial Kairós.

Cuadra, H. \& Florenzano, R. (2003). El bienestar subjetivo: hacia una psicología positiva. Revista de Psicología de la Universidad de Santiago, 12(01), 83-96.

Cueli, J. \& Morales, (2002). Psicología de los marginados. Revistas electrónicas UNAM, Los Universitarios Nueva Época, 27, 53-56.

Díaz-Morales, J. F. \& Sánchez-López, M. P. (2001). Relevancia de los estilos de personalidad y las metas en la predicción de la satisfacción vital. Anales de Psicología, 17(2), 151-158.

Díaz-Sánchez, J. (2006). Identidad, adolescencia y cultura: jóvenes secundarios en un contexto regional. Revista de Investigación Educativa, 11(29): 431-457.

Diener, E. (1994). El bienestar subjetivo. Intervención Psicosocial, 3, 67-113.

Fiorentino, M. (2008). La construcción de la resiliencia en el mejoramiento de la calidad de vida y la salud. Suma Psicológica, 15(1), 95-114.

Friedli, L. (2012). Mental Health, resilience and inequalities: a social determinants perspective. European Psychiatry, Supplement, 27, 1-1.

Garmezy, N. (1993). Resiliency and vulnerability to adverse developmental outcomes associates with poverty. American Behavioral Scientist, 34(4), 416-430.

Gómez, V., Villegas, C., Barrera, F., Cruz, J. (2007). Factores predictores de bienestar subjetivo en una muestra colombiana. Revista Latinoamericana de Psicología, 39(2), 311-325.

González, C. (2004). La psicología positiva: un cambio en nuestro enfoque patológico clásico. Liberabit, Revista de Psicología, 10, 82-88.

González, N., Valdez, J., \& Zavala, Y. (2008). Resiliencia en adolescentes mexicanos. Enseñanza e Investigación en Psicología, 13(1): 41-52.

Grotberg, E. (2003). Nuevas tendencias en resiliencia: "Resiliencia, descubriendo las propias fortalezas". México: Paidós.

Hernández-Pérez, C. (2010). La participación ciudadana en México ante la Marginación Económica. En M. Ayllon, M. Aguilar, R. \& Contreras, (Eds.), Diversidad Cultural, Ciudadanía, Política y Derecho. México: Biblioteca Virtual en Derecho, Economía y Ciencias Sociales. 
Gómez-Azcarate, Vera , Ávila , Musitu, Vega \& Dorantes I Psicodebate, 14(1) I 45-68.

Hystad, S. (2012). Exploring gender equivalence and bias in a measure of psychological hardiness. International Journal of Psychological Studies, 4(4), 69-79.

INEGI (2010). México en cifras: información nacional por entidad federativa. Instituto Nacional de Estadística y Geografía, México, D.F.

INEGI (2012). Presenta el INEGI cifras sobre el bienestar subjetivo de los mexicanos. Boletín de Prensa número 431/12 (21 de nov.). México, D.F.

Janas, M. (2002). Twenty ways to build resiliency. Intervention in School and Clinic, 38(2), 117.

Jiménez, T., Musitu, G., \& Murgui, S. (2005). Familia, apoyo social y conducta delictiva en la adolescencia: efectos directos y mediadores. Anuario de Psicología, 36(2), 181-195.

Kaztman, R. (1992). ¿Por qué los hombres son tan irresponsables?, Revista de la CEPAL, 46.

Layard, R. (2005). Happiness: Lessons from a New Science. New York: Penguin.

Martínez-Pizarro, J. (2000). Emigración internacional de jóvenes latinoamericanos y caribeños: protagonismo y vulnerabilidad. Proyecto Regional de Población CELADE-FNUAP, Serie Población y desarrollo, CEPAL, Santiago de Chile, Julio.

Montero, M. (2004). Introducción a la psicología comunitaria. Desarrollo, conceptos y procesos. Buenos Aires: Editorial Paidós.

Omar, A., Paris, L., Uribe, H., Almeida da Silva, S., \& Aguiar de Souza, M. (2011) Un modelo explicativo de la resiliencia en jóvenes y adolescentes. Psicología em Estudo, 16(2), 269-277.

Oshio, T. \& Kaobayashi, M. (2011). Area-Level Income Inequality and Individual Happiness: Evidence from Japan. Journal of Happiness Studies, 12, 633-649.

Palomar, J. \& Gómez, N. (2010). Desarrollo de una Escala de Medición de la Resiliencia con Mexicanos (RESI-M). Interdisciplinaria, 27(1), 7-22.

Palomar, J. \& Gómez, N. (2009). Plan de Desarrollo Municipal de Jiutepec 20092012. Gobierno del Municipio de Jiutepec, documento Institucional.

Programa de Naciones Unidas para el Desarrollo (2004). Informe sobre desarrollo Humano en México. México: Grupo Mundi-prensa. 
Prilletensky, I. (2004). Prólogo: validez psicopolítica: el próximo reto para la psicología comunitaria. En M. Montero (Ed.), Introducción a la psicología comunitaria. Desarrollo, conceptos y procesos. Buenos Aires: Paidós.

Rojas, M. (2004). Well-being and the complexity of Poverty. Research Paper, United Nations University, WIDER, 2004/ 29.

Romer, N., Ravitch, N., Tom, K., Merrell, K. \& Wesley, K. (2011). Gender differences in positive social-emotional functioning. Psychology in the Schools, 48(10), 958-970.

Salgado, A. (2009). Felicidad, resiliencia y optimismo en estudiantes de colegios nacionales de Lima. Liberabit, Revista de Psicología, 15(2), 133-141.

Salvia, A. (2007). Consideraciones sobre la transición a la modernidad: la exclusión social y la marginalidad económica. Un campo abierto a la investigación social y al debate político. Cátedras de la Universidad de Buenos Aires, Argentina, pp. 32.

Sen, A. (2009). The Idea of Justice. Massachusetts: Belknap Harvard Press.

Seccombe, K. (2002). "Beating the Odds" versus "Changing the Odds": Poverty, Resilience and Family Policy. Journal of Marriage \& Family, 64(2), 384-394.

Schimmel, J. (2009). Development as Happiness: The Subjective Perception of Happiness and UNDP's Analysis of Poverty, Wealth and Development. Journal of Happiness Studies, 10, 93-111.

Szloboda, P. (2008). Gratitude practices: a key to resiliency, well-being and happiness. Beginnings (American Holistic Nurses' Association), 28(1), 6-7.

Stratta, P., Capanna, C., Patriarca, S., De Catalod, S., Riccardi, I., \& Rossi, A. (2013). Resilience in adolescence: gender differences two years after the earthquake of L'Aquila. Personality \& Individual Differences, 54(3), 327-331.

Wasonga, T., Christman, D., \& Kilmer, L. (2003). Ethnicity, gender and age: predicting resilience and academic achievement among urban high school students. American Secondary Education, 32(1), 62-74. 
